- Multidisciplinary consultation regarding introduction of POS.

Results

- Music

A feeling of ownership and camaraderie promoting discussion ranging from shared memories to the spiritual needs of the present

- Tai Chi

This new skill has facilitated relaxation, breathing control and aided sleep

- POS

Its use has identified a gap in care when patients are not accessing other hospice services and ensured their needs are met.

Conclusion The exercise group has proved to be a good leveller with patients feeling confident to share experiences whilst gaining physical and emotional strength.

\section{P-141 'SMILE' DEVELOPING A SELF-MANAGEMENT PROGRAMME WITHIN A HOSPICE OUT-PATIENT SETTING}

Victoria O'Cuinneagain, Nicola Davies, Wendy Valentine. The Hospice of St Francis, Berkhamsted, UK

10.1136/bmjspcare-2017-hospice. 166

Background The hospice out-patient service offers support to patients, carers and the bereaved, we identified that clients presented with problems often poorly recognised or understood. These were significant in creating barriers and impacting on ability to self-manage and maintain independence.

Aim To educate and empower through the promotion of selfmanagement skills and coping strategies; using a series of interlinked workshops addressing commonalities of symptoms impacting on everyday living.

Method A rolling programme of workshops was designed, running bi-monthly entitled; 'SMILE'- Self- Management and Independent Living Everyday. The programme comprises four workshops;

- Fatigue management

- Sleep and relaxation

- Mindfulness and resilience

- Movement and exercise.

Results Each workshop is evaluated with positive feedback being obtained in all areas. The most notable being:

- The information and skills I have learnt have helped me now $-76 \%$ strongly agree

- The information and skills I have learnt will help me in the future $-80 \%$ strongly agree.

The Well-Being Star Outcome Measure is also used. Areas of particular influence noted were on 'symptom management', feeling positive', 'looking after yourself' and 'lifestyle'.

Feedback received:

- 'Realising sleep problems are very common and I am not alone!'

- 'giving me insight into multiple causes of fatigue'

- 'being given coping examples'.

Conclusion Patients have demonstrated a greater level of empowerment and self-management, seeking additional support. This has included requests for help returning to work, an area of support not previously in high demand. As a result of the positive outcomes there has been an increased referral rate. Attendance at sessions is variable with 'Fatigue Management' and 'Sleep and Relaxation' being most popular. The programme has been enhanced to offer individual sessions for those not suited to a group setting. Additionally increased promotion is required to encourage carers and bereaved people to recognise 'SMILE' is not a patient only programme.

\section{P-142 EIGHT POSITIVE STEPS TO WELLBEING...}

Sally List. Earl Mountbatten Hospice, Isle of Wight, UK

10.1136/bmjspcare-2017-hospice. 167

Background Self-management support can be viewed in two ways - as a portfolio of techniques and tools to help patients choose healthy behaviours, and as a fundamental transformation of the patient-caregiver relationship into a collaborative partnership. The hospice delivers a patient and carer programme to encourage self-management for patients with a range of life-limiting conditions. The planning of the programme was carried out with the input of the hospice User Forum, with the scoping of existing groups delivered by hospices across England. The first programme was initially piloted to identify the key sessions and format of the programme. Self-management programmes for patients with long-term conditions have been developed and researched over the past decade. There is limited research concerning these for adults with life-limiting conditions.

Aims Supporting adults with any life-limiting illness - offering information and support to help people manage their illness along with carers, family and friends who support them.

Methods People are invited to attend one or more sessions, which include eating well, living with fatigue, encouraging exercise, coping with being breathless, protecting skin, information about welfare benefits and how to cope with medical and care changes. The hour-long sessions are facilitated by an expert in the relevant field either from the hospice or partner organisations, including Age UK and the NHS Trust.

Results First programme - 31 attendees; second programme 53 attendees. Examples of participant feedback - 'I feel rejuvenated'; 'I close my flat door and come here for positive input'; 'I had no idea of the range of moulds for the pureed foods I need to have'.

Conclusions Voluntary participation, positive group dynamics, strong but flexible facilitators and active learning techniques underpin the overwhelmingly positive experiences of the sessions now being rolled out across the year.

\section{P-143 ENABLING NOT DISABLING}

Becky McGregor, Elizabeth Arnold. Earl Mountbatten Hospice, Isle of Wight, UK

10.1136/bmjspcare-2017-hospice. 168

Introduction A successful funding application to the St James's Place Foundation through Hospice UK has seen the commencement of a project focusing on enabling rather than disabling people; blending compassion and care with a rehabilitative and enabling approach.

Aims of the Project The project aims to shift the culture from one of 'over caring' within hospice services, to one that is working for and with people to determine what living well 
looks like for them individually. The project aims to utilise a collaborative, interdisciplinary, and whole team approach.

Approach Used The project has taken a multi-faceted approach:

Leadership

- Appoint a project lead

- Set up a multi-professional task and finish group

- Champion and role model a different way of thinking.

Training

- Delivery of training sessions from ward to Board

- Develop core enablement and rehabilitation competencies for assistant staff

- Incorporate rehabilitation and enablement approach into staff and volunteer induction.

Service development

- Implementation of a programme of circuit exercise in the gym

- Development of 'What Matters to Me' documentation

- Implementation of a self-management 'Positive Steps to Well-being' programme

- Development of a visual manual handling plan for patients on the Inpatient Unit

- Pilot mirroring package of care on the Inpatient Unit prior to discharge home

- Development of a new volunteer community enablement assistant role.

Organisational structure and process

- Staff job descriptions/volunteer role profiles to reflect rehabilitation and enablement

- Incorporate reflection and evidence of rehabilitative palliative care practice in the appraisal process

- New healthcare assistants shadow the Allied Health Professions team to learn rehabilitative skills in practice.

Outcomes On completion of the project, the hospice aims to increase our total rehabilitative palliative care score to over 80/111 from 29/111 in August 2015 and 51/111 in August 2016 (Tiberini \& Richardson, 2015).

Conclusion Effective implementation of an enabling approach has required a stepped approach, an understanding of organisational readiness, and key cultural barriers.

\section{P-144 WHOSE CHOICE IS IT ANYWAY?}

Maureen Howe, Anthony Walsh, Jackie Green, Margaret Hollinshead. St Catherine's Hospice, Lancashire, UK

\subsection{6/bmjspcare-2017-hospice. 169}

Background The rehabilitation team consists of two part-time physiotherapists, totalling just over one whole-time equivalent. Most of the time they work single-handedly. Whilst reviewing their roles, the Rehabilitation Team identified two main concerns.

When patients want to do an activity that requires more than one person to facilitate it, the physiotherapist would have to wait for another member of staff to be available.

There were missed opportunities for some patients for example; those that were independent and in the hospice for symptom control.

Aims To increase the rehabilitation resource.

Every patient to have options for activities, and enable them to continue these outside of the hospice environment.
Method

- Patient focus groups met over a three month period to determine which patient needs were not being met

- Applied to St James's Place Foundation for funding of $£ 35000$ to establish a training programme for volunteers

- Recruited and trained a team of volunteers, two people per week day.

Results Nine volunteers trained - induction, full moving and handling, some had fatigue, anxiety and breathlessness management training. Each volunteer has an average of 5 contacts per day. The rehabilitation team now includes trained volunteers. They support the nursing and rehabilitation teams to give patients an increased choice of activities and provide another pair of hands for mobility or functional assessments.

'Jonny was really good with my Mum this morning, showing her how to get out of bed and spending time with her in Day Therapy' - Daughter of the patient

'Having nursing assistants with enhanced skills and knowledge working alongside volunteers has had such a positive impact on the team's ability to carry out the necessary assessments to meet our patients' needs without delay.' - Sister InPatient Unit

Future Developments

- Consideration of a seven day service

- Further training of the volunteer team.

\section{P-145 LIVING WELL WITH HEART FAILURE: A CREATIVE PARTNERSHIP APPROACH}

Sian Cole. Longfield, Gloucestershire, UK

10.1136/bmjspcare-2017-hospice. 170

Background One in 15 people aged $75-84$ in the UK have some degree of heart failure. Longfield working collaboratively with the Heart Failure Clinical Nurse Specialist team developed and piloted a six-week rehabilitative heart failure programme.

Aims To develop, pilot and evaluate a heart failure programme. The programme used a combined clinical and creative approach, bringing together the skills of Heart Failure CNS and Creative Therapists. The objectives of the programme were to increase exercise tolerance, knowledge and understanding of heart failure, improve feelings of well-being and support patients to achieve better health outcomes, such as decrease in medical interventions.

Methods The six-week programme had three main components: clinical, creative therapy and exercise in the form of Tai Chi.

Topics covered during the programme included managing breathlessness and fatigue, advance care planning, nutrition, weight loss, medication, mood and stress management. Sessions concluded with an exercise component that consisted of Tai Chi.

Results Two programmes were delivered with 13 attendees in total. In addition we ran a taster/introductory session to which carers and participants were invited, to give them an overview of the programme. Qualitative and quantitative patient feedback was gathered at the beginning and at the end of both programmes. Feedback was positive with respondents feeling more able to engage in exercise and increased confidence in managing symptoms and medication. In the second pilot 